\title{
Exact Inverse Operator on Field Equations
}

\author{
Edwin Eugene Klingman (1) \\ Cybernetic Micro Systems Inc., San Gregorio, CA, USA \\ Email: klingman@geneman.com
}

How to cite this paper: Klingman, E.E. (2020) Exact Inverse Operator on Field Equations. Journal of Applied Mathematics and Physics, 8, 2213-2222.

https://doi.org/10.4236/jamp.2020.810166

Received: September 21, 2020

Accepted: October 24, 2020

Published: October 27, 2020

Copyright (๑) 2020 by author(s) and Scientific Research Publishing Inc. This work is licensed under the Creative Commons Attribution International License (CC BY 4.0).

http://creativecommons.org/licenses/by/4.0/

\begin{abstract}
Differential equations of electromagnetic and similar physical fields are generally solved via antiderivative Green's functions involving integration over a region and its boundary. Research on the Kasner metric reveals a variable boundary deemed inappropriate for standard anti-derivatives, suggesting the need for an alternative solution technique. In this work I derive such a solution and prove its existence, based on circulation equations in which the curl of the field is induced by source current density and possibly changes in associated fields. We present an anti-curl operator that is believed novel and we prove that it solves for the field without integration required.
\end{abstract}

\section{Keywords}

Anti-Derivative, Anti-Curl Operator, Maxwell's Equations, Geometric Calculus, Kasner Metric, Green's Function, Biot-Savart Operator

\section{Introduction}

Nonlinear field equations are generally impossible of solution-the few existing (Schwarzschild, Kerr) being well-known. These known solutions are static and expressed in spherical coordinates with boundaries at infinity. The Kasner solution, treated by Vishwakarma [1], is dynamic with dynamic boundaries and does not fit well into the static approach. Even physical interpretation of the exact solution has been largely nonexistent. For this reason an alternative approach was sought. The goals of this approach were:

1) A linear formulation that avoids the nonlinearity of Einstein's field equations.

2) A "boundaryless" solution, eliminating the usual anti-derivative-based approach.

3) A physical interpretation that makes sense of the new solution, and

4) An existence proof for the new solution technique.

This paper primarily treats the last problem. 


\section{Relevant Mathematical Background}

Physicists are familiar with Maxwell's vector-based equations describing electric and magnetic fields, $\boldsymbol{E}$ and $\boldsymbol{B}$ respectively, in terms of changes over space and time, based on differential operators $\nabla$ and $\partial_{t}$ and sources of the fields Most are familiar with the tensor formulation:

$$
F_{\mu \nu}=\left|\begin{array}{cccc}
0 & -E_{x} & -E_{y} & -E_{z} \\
E_{x} & 0 & B_{z} & -B_{y} \\
E_{y} & -B_{z} & 0 & B_{x} \\
E_{z} & B_{y} & -B_{x} & 0
\end{array}\right|
$$

where

$$
F_{\mu \nu}=\partial_{\mu} A_{-}-\partial_{\nu} A_{\mu}, \quad E_{n}=-\left(\partial_{0} A_{n}-\partial_{n} A_{0}\right), \quad \boldsymbol{B}=\nabla \times \boldsymbol{A}
$$

A growing number of physicists are familiar with Hestenes' geometric algebra [2] derived from Clifford and Grassmann algebras, in which every entity has both an algebraic and a geometric interpretation. For classical 3D physics the entities consists of scalars, vectors, bivectors, and trivectors/pseudoscalars. In this formulation the electromagnetic field is a multivector [3],

$$
F(x, t)=\boldsymbol{E}(\boldsymbol{x}, t)+i \boldsymbol{B}(\boldsymbol{x}, t)
$$

where $\boldsymbol{E}$ is the usual electric field vector and the magnetic field is a bivector, a directed 2D-planar entity representing rotation of one vector into another, and $i$ is the pseudoscalar or dual operator that maps bivector $\boldsymbol{B}$ into its vector dual.

Magnetic field vectors do not behave like vectors under reflection, they are not really vectors; axial vectors are not encoded in vector space formalism [4]. However such behavior is encoded in bivector formalism, therefore the geometric algebra function better represents electromagnetic fields. A key geometric algebra entity is the geometric product of two vectors, $u$ and $v$ :

$$
u v=u \cdot v+u \wedge v
$$

where outer product $\boldsymbol{u} \wedge \boldsymbol{v}$ does not exist in vector calculus but is dual [5] of the cross product

$$
\boldsymbol{u} \wedge \boldsymbol{v}=i \boldsymbol{u} \times \boldsymbol{v}
$$

If we let the first vector in Equation (4) be the vector derivative $\nabla$ we obtain

$$
\nabla \boldsymbol{f}=\nabla \cdot \boldsymbol{f}+\nabla \wedge \boldsymbol{f}
$$

where $\nabla f$ is the gradient, $\nabla \cdot f$ is the divergence, and $\nabla \wedge f$ is the curl of $f$. Hence in geometric calculus we have the unique relation

$$
\text { gradient }=\text { divergence }+ \text { curl }
$$

which has no analog in vector calculus. Relevant identities for the outer product are:

$$
\begin{aligned}
& \nabla \wedge(\nabla \wedge f)=0 \text { - curl of a curl is zero } \\
& \nabla \wedge(\nabla f)=0 \text {-curl of gradient of scalar field is zero } \\
& \nabla \cdot(\nabla \cdot f)=0 \text {-divergence of a divergence is zero }
\end{aligned}
$$


Geometric calculus generalizes vector calculus and Cartan's calculus of differential forms. The elementary combination of inner and outer products,

$\boldsymbol{u} \boldsymbol{v}=\boldsymbol{u} \cdot \boldsymbol{v}+\boldsymbol{u} \wedge \boldsymbol{v}$ cannot be expressed with differential forms [6]; such limitations are addressed by extending the formalism with the theory of fiber bundles. Forms are insufficient for most applications and must be supplemented by other algebraic systems such as matrices or tensors. In geometric calculus spinors, tensors, linear transformations and differential forms are developed in a unified mathematical system. Forms have no analog of $\nabla f=\nabla \cdot f+\nabla \wedge f$ wherein $\nabla \cdot f$ and $\nabla \wedge f$ are two distinct parts of the single fundamental quantity, coderivative $\nabla f$, that makes it possible to reduce Maxwell's eqns to a single equation:

$$
\nabla \boldsymbol{F}=\boldsymbol{J} .
$$

When $\nabla f=\partial f$ it can be directly solved for $f$, whereas $\nabla \cdot f$ and $\nabla \wedge f$ cannot, because each is only part of the derivative of $\nabla f$. Finally, geometric calculus, whose fundamental theorem on $m$-dimensional manifolds

$$
\int_{M} \mathrm{~d}^{m} x \partial F=\oint_{\partial M} \mathrm{~d}^{m-1} x F
$$

provides a unified mathematical tool for physics; Equation (9) subsumes Gauss's theorem, Stoke's theorem, Green's theorem, and the Cauchy integral formula, as well as generalizing vector calculus. Although the results of this paper are intended to augment geometric calculus, we will limit our proofs to vector calculus. Arthur's geometric algebra treatment of Maxwell's equations provides a $(3+1) \mathrm{D}$ formulation of 3-space vector and 1-time scalar in detail, and then develops the $4 \mathrm{D}$ formulation in which time is promoted from scalar to vector.

\section{Biot-Savart Inverse Operator and Laplacian}

Much of the literature on the use of Biot-Savart operators and Green's function-based inverse operation deals with the scalar Laplacian in the Poisson equation,

$$
\Delta \phi=\rho
$$

where Laplacian $\Delta=\nabla \cdot \nabla$. The inverse operator yields

$$
\phi(x)=\left[\Delta^{-1}\right] \rho(x)=\int_{\mathfrak{R}^{3}} \frac{\rho(y)}{4 \pi\|x-y\|} \mathrm{d}^{3} y
$$

where $\Delta$ has right inverse $\left[\Delta^{-1}\right]$ with integral kernel $\phi(x, y)=A(d(x, y))$ depending on the distance only. In [7] the right inverse for the Laplace operator on forms is constructed and proved based on an integral kernal determined by function $A$ which they prove to always exist. They further show that a right inverse $\Delta^{-1}$ of the Laplacian operator on differential forms provides a right inverse to the Cartan differential $d$ and co-differential $d^{*}$ simultaneously. The formalism yields a Biot-Savart operator motivated by the analogy of the equations with Maxwell equations for a magnetic field $\boldsymbol{B}$ caused by stationary cur- 
rent $J$. For Laplacian $\Delta=d d^{*}+d^{*} d$ they derive $\Delta^{-1}$ as an integral kernal determined by function $A$.

\section{Real Physical Field Solutions}

Real physical fields are sourced by distributions or current flows. The differential equation describing field dependence on source current density has the form

$$
\partial \boldsymbol{f}=\boldsymbol{j}
$$

which is formally solved via the inverse differential operator

$$
\boldsymbol{f}=\partial^{-1} \boldsymbol{j} .
$$

The inverse operation $\partial^{-1}$ is satisfied by the existence of the Green's function anti-derivative method of solution for physical field equations. The Dirac delta distribution-based technique provides field solutions in terms of boundary conditions. If $i$ is the unit pseudo-vector and $M$ is an $m$-dimensional smooth oriented vector manifold with piecewise smooth boundary $\partial M$ then

$$
\boldsymbol{f}=\frac{1}{(-1)^{m} i}\left[\int_{M} g\left(x, x^{\prime}\right) \mathrm{d} X\left(x^{\prime}\right) \partial^{\prime} f\left(x^{\prime}\right)+\int_{\partial M} g\left(x, x^{\prime}\right) \mathrm{d} S\left(x^{\prime}\right) f\left(x^{\prime}\right)\right]
$$

where Green's function $g\left(x, x^{\prime}\right)=\frac{g\left(x-x^{\prime}\right)}{\left|x-x^{\prime}\right|^{3}}$. This is the general solution corresponding to anti-derivative $\partial^{-1}$ for equation $\partial \boldsymbol{f}=\boldsymbol{j}$. Explicitly, values of $\partial \boldsymbol{f}$ at interior points of $M$ are determined by values of $\boldsymbol{f}$ on boundary $\partial M$, as well as by values of $\boldsymbol{f}$ inside $M$.

\section{Discrete Operator}

It is the purpose of this paper to establish the discrete operator analog of the anti-derivative-based $\partial^{-1} \Rightarrow \int_{M}+\int_{\partial M}$. In place of integrating over every point in the region, as well as all points in the boundary of the manifold, the preferred formulation would be $\partial^{-1} \partial \Rightarrow\left|\begin{array}{lll}1 & 0 & 0 \\ 0 & 1 & 0 \\ 0 & 0 & 1\end{array}\right|$.

Consider two situations; the electric field $\boldsymbol{E}$ induced by a static charge density $\rho(\boldsymbol{x})$ and the magnetic field $\boldsymbol{B}$ induced by moving charge density $\rho(\boldsymbol{x}) \boldsymbol{v}$. We consider each separately:

$$
\nabla \boldsymbol{E}=\nabla \cdot \boldsymbol{E}+\nabla \wedge \boldsymbol{E}=\rho
$$

If $\boldsymbol{B}$ is not changing, $\nabla \wedge \boldsymbol{E}=0$ and $\boldsymbol{\nabla} \cdot \boldsymbol{E}=\rho$, hence $\boldsymbol{E}=\nabla^{-1} \rho$ where $\nabla^{-1}$ is an integral (anti-derivative) operator determined by a Green's function. An interesting case [8] is for $\rho(\boldsymbol{x})$ with $2 \mathrm{D}$ symmetry in $2 \mathrm{D}$ region $\mathfrak{R}$ with boundary $\partial \Re$ :

$$
\boldsymbol{E}(\boldsymbol{x})=\frac{1}{2 \pi} \int_{\Re} \mathrm{d}^{2} x^{\prime} \frac{\rho\left(x^{\prime}\right)}{\boldsymbol{x}-\boldsymbol{x}^{\prime}}+\frac{1}{2 \pi i} \oint_{\partial \mathfrak{R}} \frac{\boldsymbol{E}(\boldsymbol{x}) \cdots \mathrm{d} x^{\prime}}{\boldsymbol{x}-\boldsymbol{x}^{\prime}}
$$


In the absence of sources the first integral vanishes and the field within $\Re$ is given by the line integral of its value over the boundary $\partial \Re$, which can be seen to be the Cauchy integral formula after suitable change of variables.

We next consider the coderivative of the magnetic field

$$
\nabla \boldsymbol{B}=\nabla \cdot \boldsymbol{B}+\nabla \wedge \boldsymbol{B}=\rho \boldsymbol{v} .
$$

With gauge field $\boldsymbol{A}$ and $\boldsymbol{B}=\boldsymbol{\nabla} \times \boldsymbol{A}$ and vector identity $\boldsymbol{\nabla} \cdot \boldsymbol{\nabla} \times \boldsymbol{A}=0$, this reduces to $\nabla \times \boldsymbol{B}=\boldsymbol{j}$, the prototype equation for our theorem and proof. This choice is compatible with the universal equation describing the field circulation induced by source current density:

$$
\nabla \times f=j
$$

where source current density $\boldsymbol{j}=\rho \boldsymbol{v}$ with $\rho=q / r^{3}, q$ is source charge and $v$ is flow velocity.

"The [geometric algebra] vector derivative $\nabla$ actually has an inverse, in complete contrast to traditional $3 D$ vector analysis where only the gradient has a definite inverse and the curl and divergence do not."

Despite this definite statement, we search for a solution of the form symbolically represented as

$$
f=\frac{j}{(\nabla \times)}
$$

To derive this solution we need to show that the inverse curl operator exists:

$$
(\nabla \times)^{-1} \equiv \frac{1}{(\nabla \times)}
$$

The remainder of this paper establishes the existence of the inverse curl operator.

\section{The Uncurl Operator Theorem}

$$
\text { Theorem I } \exists(\nabla \times)^{-1} \ni \nabla \times \boldsymbol{f}=\boldsymbol{j} \Rightarrow \boldsymbol{f}=(\nabla \times)^{-1} \boldsymbol{j}
$$

There is an anti-curl operator $(\nabla \times)^{-1}$ with inverse curl property such that, applied to differential equation $\nabla \times \boldsymbol{f}=\boldsymbol{j}$ solves for field vector $\boldsymbol{f}=(\nabla \times)^{-1} \boldsymbol{j}$.

Our purpose in this paper is to present a $3 \times 3$-operator-based inverse $(\nabla \times)^{-1}$ which will allow solution at specific field points $\boldsymbol{r}$ distant from the source current density. The solution is generally simpler, more intuitive, and avoids integration over boundaries of the vector manifold. It also allows mathematical solutions in situations with undefined or dynamic boundaries as seen in the Kasner metric-based exact solution to Einstein's field equations of general relativity [9].

Of course any way of finding a good solution is legitimate; typically guessing based on intuition or analogy, however contained within the beauty of mathematical physics is the fact that the physical world often suggests the answer. In the case of electrodynamic fields we know the relation of the field to the source current density. We know that $\boldsymbol{f}=\boldsymbol{r} \times \boldsymbol{j}$, with unit scale factor, therefore we 
solve $\nabla \times \boldsymbol{f}=\boldsymbol{j}$ for $\boldsymbol{f}$ by operating with anti-curl operator $(\nabla \times)^{-1}$ on both sides of

$$
(\nabla \times)^{-1}(\nabla \times) \boldsymbol{f}=(\nabla \times)^{-1} \boldsymbol{j}
$$

Given

$$
\boldsymbol{f}=\boldsymbol{r} \times \mathbf{j}
$$

we have the suggested association $(\nabla \times)^{-1}=(\boldsymbol{r} \times)$. We will prove that use of radial operator $(\boldsymbol{r} \times)$ in place of inverse curl operator $(\nabla \times)^{-1}$ satisfies Equation (21) above.

In the theorem $(\nabla \times)^{-1}(\nabla \times) \boldsymbol{f}=(\nabla \times)^{-1} \boldsymbol{j}$ where $\boldsymbol{j}=\left\{\frac{j_{x}}{r^{3}}, \frac{j_{y}}{r^{3}}, \frac{j_{z}}{r^{3}}\right\}$ we stipulate that the source current density $\rho=s / r^{3}$ is defined using the same volume radius $r=|\boldsymbol{r}|$ as is used in the inverse curl operator $(\boldsymbol{r} \times)$. This is not an arbitrary condition but is implied by the physics $\boldsymbol{B}(\boldsymbol{r})=(\boldsymbol{r} \times \boldsymbol{j}) / r^{3}$ and is seen to be compatible with the Green's function-based anti-derivative $g\left(x, x^{\prime}\right)=\frac{g\left(x-x^{\prime}\right)}{\left|x-x^{\prime}\right|^{3}}$. Physically this traces to Birkhoff's theorem, a generalization of Newton's Shell theorem in which the source inside an isotropic homogeneous spherical shell can be treated as concentrated at the center of the shell and all source outside the shell can be ignored as its contributions cancel.

Hypothesis: The inverse curl operator $(\nabla \times)^{-1}$ is the radius operator $(\boldsymbol{r} \times)$.

The plan of our proof is to prove that $(\nabla \times)^{-1}(\nabla \times) f=(\nabla \times)^{-1} \boldsymbol{j}$ where $(\nabla \times)^{-1}=(\boldsymbol{r} \times)$. We explicitly expand the expression to obtain the terms $\boldsymbol{r} \times \boldsymbol{\nabla} \times \boldsymbol{f}$, where we replace the source current density $\boldsymbol{j}$ with the completely equivalent momentum density $\boldsymbol{p}=\left\{\frac{p x}{r^{3}}, \frac{p y}{r^{3}}, \frac{p z}{r^{3}}\right\}$. Here and in the following, the expression $j x \equiv j_{x}$ and $p x \equiv p_{x}$.

$$
\boldsymbol{r} \times \nabla \times \boldsymbol{f}=\boldsymbol{f}=\boldsymbol{r} \times \boldsymbol{p}
$$

The expansion of this equation is below:

$$
\begin{aligned}
& \left\{\frac{p z x^{2} y}{\left(r^{2}\right)^{\frac{5}{2}}}+\frac{p z y^{3}}{\left(r^{2}\right)^{\frac{5}{2}}}-\frac{p y x^{2} z}{\left(r^{2}\right)^{\frac{5}{2}}}-\frac{p y y^{2} z}{\left(r^{2}\right)^{\frac{5}{2}}}+\frac{p z y z^{2}}{\left(r^{2}\right)^{\frac{5}{2}}}-\frac{p y z^{3}}{\left(r^{2}\right)^{\frac{5}{2}}},\right. \\
& -\frac{p z x^{3}}{\left(r^{2}\right)^{\frac{5}{2}}}-\frac{p z x y^{2}}{\left(r^{2}\right)^{\frac{5}{2}}}+\frac{p x x^{2} z}{\left(r^{2}\right)^{\frac{5}{2}}}+\frac{p x y^{2} z}{\left(r^{2}\right)^{\frac{5}{2}}}-\frac{p z x z^{2}}{\left(r^{2}\right)^{\frac{5}{2}}}+\frac{p x z^{3}}{\left(r^{2}\right)^{\frac{5}{2}}}, \\
& \left.\frac{p y x^{3}}{\left(r^{2}\right)^{\frac{5}{2}}}-\frac{p x x^{2} y}{\left(r^{2}\right)^{\frac{5}{2}}}+\frac{p y x y^{2}}{\left(r^{2}\right)^{\frac{5}{2}}}-\frac{p x y^{3}}{\left(r^{2}\right)^{\frac{5}{2}}}+\frac{p y x z^{2}}{\left(r^{2}\right)^{\frac{5}{2}}}-\frac{p x y z^{2}}{\left(r^{2}\right)^{\frac{5}{2}}}\right\} \\
& =\left\{\frac{p z y}{\left(r^{2}\right)^{3 / 2}}-\frac{p y z}{\left(r^{2}\right)^{3 / 2}},-\frac{p z x}{\left(r^{2}\right)^{3 / 2}}+\frac{p x z}{\left(r^{2}\right)^{3 / 2}}, \frac{p y x}{\left(r^{2}\right)^{3 / 2}}-\frac{p x y}{\left(r^{2}\right)^{3 / 2}}\right\}
\end{aligned}
$$


Proposition: Terms on the left-hand side of $\boldsymbol{r} \times \boldsymbol{\nabla} \times \boldsymbol{f}=\boldsymbol{f}$ are organized into three expressions, the $x$-, $y$-, and $z$-components of the field vector $f$ where $\boldsymbol{f}=\boldsymbol{r} \times \boldsymbol{j}$ and $\boldsymbol{p}$ is the source current density $\boldsymbol{p}=\left\{\frac{p x}{r^{3}}, \frac{p y}{r^{3}}, \frac{p z}{r^{3}}\right\}$ that replaces current density $\boldsymbol{j}$ for consistency with other problems.

lemma 1. $(\boldsymbol{r} \times \boldsymbol{\nabla} \times \boldsymbol{f})_{x}=(\boldsymbol{r} \times \boldsymbol{p})_{x}$

We regroup the terms in the $\mathrm{x}$-component of the left hand side as follows:

$$
\left\{\frac{p z y x^{2}}{r^{5}}+\frac{p z y y^{2}}{r^{5}}+\frac{p z y z^{2}}{r^{5}}\right\}=\left\{\frac{p z y r^{2}}{r^{5}}\right\}=\frac{p z y}{r^{3}} .
$$

Similarly we regroup the remaining terms in the $\mathrm{x}$-component of the left hand side as follows:

$$
-\left\{\frac{p y z x^{2}}{r^{5}}+\frac{p y z y^{2}}{r^{5}}+\frac{p y z z^{2}}{r^{5}}\right\}=-\left\{\frac{p y z r^{2}}{r^{5}}\right\}=-\frac{p y z}{r^{3}} .
$$

Grouping these two terms we see that they are equal to the $\mathrm{x}$-component of the right hand side:

$$
(\boldsymbol{r} \times \nabla \times \boldsymbol{f})_{x}=\left\{\frac{p z y}{r^{3}}-\frac{p y z}{r^{3}}\right\}=\left\{\frac{p z y}{r^{3}}-\frac{p y z}{r^{3}}\right\}=(\boldsymbol{r} \times \boldsymbol{p})_{x}=\boldsymbol{f}_{x}
$$

and similarly for $y$ - and $z$-components. The inverse curl operator $(\nabla \times)^{-1}$ is radius operator $(\boldsymbol{r} \times)$.

Theorem II

$$
\exists(\nabla \times)^{-1} \ni(\nabla \times)(\nabla \times)^{-1} \boldsymbol{j}=\boldsymbol{j} \Rightarrow \nabla \times \boldsymbol{f}=\boldsymbol{j} \Leftrightarrow \boldsymbol{f}=(\nabla \times)^{-1} \boldsymbol{j} \wedge(\boldsymbol{r} \perp \boldsymbol{j})
$$

There is an anti-curl operator $(\nabla \times)^{-1}$ with inverse curl property such that, applied to source current density $\boldsymbol{j}$ satisfies differential equation $\nabla \times \boldsymbol{f}=\boldsymbol{j}$ when field vector $\boldsymbol{f}=(\nabla \times)^{-1} \boldsymbol{j}$ and $\boldsymbol{r}$ is perpendicular to $\boldsymbol{j}, \boldsymbol{r} \perp \boldsymbol{j}$.

As before we substitute the momentum density $\boldsymbol{p}=\left\{\frac{p x}{r^{3}}, \frac{p y}{r^{3}}, \frac{p z}{r^{3}}\right\} \equiv\left\{\frac{p_{x}}{r^{3}}, \frac{p_{y}}{r^{3}}, \frac{p_{z}}{r^{3}}\right\}$ for current density $\boldsymbol{j}$ with no mathematical effect.

$$
\nabla \times \boldsymbol{r} \times \boldsymbol{p}=\left(\begin{array}{c}
-\frac{2 p x}{\left(r^{2}\right)^{3 / 2}}-\frac{3 p y x y}{\left(r^{2}\right)^{5 / 2}}+\frac{3 p x y^{2}}{\left(r^{2}\right)^{5 / 2}}-\frac{3 p z x z}{\left(r^{2}\right)^{5 / 2}}+\frac{3 p x z^{2}}{\left(r^{2}\right)^{5 / 2}} \\
-\frac{2 p y}{\left(r^{2}\right)^{3 / 2}}+\frac{3 p y x^{2}}{\left(r^{2}\right)^{5 / 2}}-\frac{3 p x x y}{\left(r^{2}\right)^{5 / 2}}-\frac{3 p z y z}{\left(r^{2}\right)^{5 / 2}}+\frac{3 p y z^{2}}{\left(r^{2}\right)^{5 / 2}} \\
-\frac{2 p z}{\left(r^{2}\right)^{3 / 2}}+\frac{3 p z x^{2}}{\left(r^{2}\right)^{5 / 2}}+\frac{3 p z y^{2}}{\left(r^{2}\right)^{5 / 2}}-\frac{3 p x x z}{\left(r^{2}\right)^{5 / 2}}-\frac{3 p y y z}{\left(r^{2}\right)^{5 / 2}}
\end{array}\right)=\boldsymbol{p}
$$

Proposition: The terms on the left-hand side of $\nabla \times \boldsymbol{r} \times \boldsymbol{p}=\boldsymbol{p}$ can be organized into three terms, $-2 \boldsymbol{p},+3 \boldsymbol{p}$, and $-3 \boldsymbol{r}(\boldsymbol{p} \cdot \boldsymbol{r}) / r^{2}$ where $\boldsymbol{p}$ is the source current density $\boldsymbol{p}=\left\{\frac{p x}{r^{3}}, \frac{p y}{r^{3}}, \frac{p z}{r^{3}}\right\}$. 
lemma 1. $-2 \boldsymbol{p} \in(\nabla \times(\boldsymbol{r} \times(\boldsymbol{p}))) \equiv(\nabla \times)(\nabla \times)^{-1} \boldsymbol{p}$

By visual inspection, the first column contains terms

$$
\left\{\frac{-2 p x}{r^{3}}, \frac{-2 p y}{r^{3}}, \frac{-2 p z}{r^{3}}\right\} \equiv-2 \boldsymbol{p} .
$$

lemma 2. $+3 \boldsymbol{p} \in \boldsymbol{\nabla} \times \boldsymbol{r} \times \boldsymbol{p}$

After removing the $-2 \boldsymbol{p}$ terms we find terms of the form $\frac{3 p x y^{2}}{r^{5}}$ plus $\frac{3 p x z^{2}}{r^{5}}$ but the $\frac{3 p x x^{2}}{r^{5}}$ term is missing; therefore we add and subtract $\frac{3 p x x^{2}}{r^{5}}$ to the $\mathrm{x}$-component terms. Combining these three positive terms we substitute $x^{2}+y^{2}+z^{2}=r^{2}$ and obtain $\left(3 p x r^{2} / r^{5}\right)=3 p x / r^{3}$. The same procedure applied to the $y$-and $z$-components leads to

$$
\left\{\frac{3 p x}{r^{3}}, \frac{3 p y}{r^{3}}, \frac{3 p z}{r^{3}}\right\}=+3 p .
$$

lemma 3. The remaining terms in the $x$-component are

$$
-\frac{3 p y x y}{r^{5}}-\frac{3 p z x z}{r^{5}}-\frac{3 p x x x}{r^{5}}=\frac{-3 x(\boldsymbol{p} \cdot \boldsymbol{r})}{r^{2}}
$$

The $y$ - and $z$-component terms similarly yield $\frac{-3 y(\boldsymbol{p} \cdot \boldsymbol{r})}{r^{2}}$ and $\frac{-3 z(\boldsymbol{p} \cdot \boldsymbol{r})}{r^{2}}$. Summing these three terms we obtain

$$
\frac{-3 \boldsymbol{r}(\boldsymbol{p} \cdot \boldsymbol{r})}{r^{2}}
$$

lemma 4. The result of the above steps yields $\boldsymbol{r} \perp \boldsymbol{p}$ : We have obtained equation

$$
-2 \boldsymbol{p}+3 \boldsymbol{p}-\frac{3 \boldsymbol{r}(\boldsymbol{p} \cdot \boldsymbol{r})}{r^{2}}=\boldsymbol{p} \Leftrightarrow \boldsymbol{p} \cdot \boldsymbol{r}=0
$$

which is true if and only if $-\frac{3 \boldsymbol{r}(\boldsymbol{p} \cdot \boldsymbol{r})}{r^{2}}=0$. By definition $\boldsymbol{p} \neq 0$ since this term represents the source current density or momentum density that induces a circulation in the field in question. As source current density does not have meaning at a point, distance from this nonexistent point cannot be zero hence $r \neq 0$. The remaining possibility is $\boldsymbol{p} \cdot \boldsymbol{r}=0$, whence $\boldsymbol{r} \perp \boldsymbol{p}$.

From Theorem I $(\nabla \times)^{-1}=(\boldsymbol{r} \times)$ therefore $(\nabla \times)(\nabla \times)^{-1} \boldsymbol{p}=\boldsymbol{p}$ implies $(\nabla \times)(\boldsymbol{r} \times) \boldsymbol{p}=\boldsymbol{p}$ iff $\boldsymbol{r} \perp \boldsymbol{p}$. Thus if we define $\boldsymbol{f}=\boldsymbol{r} \times \boldsymbol{p}$ we obtain $\nabla \times \boldsymbol{f}=\boldsymbol{p}$.

\section{Results}

We hypothesized the existence of an exact un-curl operator, $(\nabla \times)^{-1}$ which solves the field equation $\nabla \times \boldsymbol{f}=\boldsymbol{p}$ yielding $\boldsymbol{f}=(\nabla \times)^{-1} \boldsymbol{p}$ and we have shown that this matches the known solution $\boldsymbol{f}=\boldsymbol{r} \times \boldsymbol{p}$. Operators require operands to operate upon; equivalence relations are summarized 


$$
\begin{aligned}
& (\nabla \times)^{-1}(\nabla \times) \boldsymbol{f} \Rightarrow\left(\begin{array}{lll}
1 & 0 & 0 \\
0 & 1 & 0 \\
0 & 0 & 1
\end{array}\right) \boldsymbol{f} \\
& (\nabla \times)(\nabla \times)^{-1} \boldsymbol{p} \Rightarrow\left(\begin{array}{lll}
1 & 0 & 0 \\
0 & 1 & 0 \\
0 & 0 & 1
\end{array}\right) \boldsymbol{p}
\end{aligned}
$$

We proved these operator relations in terms of the field equations:

$$
(\boldsymbol{r} \times \boldsymbol{\nabla} \times \boldsymbol{f})=\boldsymbol{f}, \quad(\boldsymbol{\nabla} \times \boldsymbol{r} \times \boldsymbol{p})=\boldsymbol{p} .
$$

\section{Discussion of Results}

The inverse operation on field equations typically derives an anti-derivativebased solution for the field based on integrals of the field derivative for every point within the bounded region and an integral evaluating all points on the boundary of the region. Contrast the two solutions for the magnetic field at $\boldsymbol{r}$ from a source current; the Biot-Savart law [10] for a steady current $\boldsymbol{J}$ inside a region $\Omega \subset \mathfrak{R}^{3}$, where $\Omega$ could represent a curve, surface, or volume; the magnetic field associated to $\boldsymbol{J}$ is

$$
\boldsymbol{B}(\boldsymbol{r})=\boldsymbol{r} \times \boldsymbol{j} \text { versus } \boldsymbol{B}(\boldsymbol{r})=\int_{\Omega} J(x) \times \frac{\boldsymbol{r}-\boldsymbol{r}^{\prime}}{\left|\boldsymbol{r}-\boldsymbol{r}^{\prime}\right|^{3}} \mathrm{~d} r^{\prime} .
$$

If we wish to integrate over a length of current path, the anti-derivative is preferable. The inverse operation on field equations derived herein is significantly simpler and derives the value of the field at a point a distance $\boldsymbol{r}$ from source current density. Much effort has been spent on generalizing Green's function results in curved space; nevertheless; there exist gravitomagnetic problems in metric space wherein the boundary varies dynamically, and flat space is a realistic assumption. The anti-curl operator enables new approaches to the physics of fields that are effectively unobtainable using Green's function-based anti-derivative inverses.

\section{Conflicts of Interest}

The author declares no conflicts of interest regarding the publication of this paper.

\section{References}

[1] Vishwakarma, R. Gravity of $R^{\mu v}=0$ : A New Paradigm in GR. arXiv:1206.2795v2.

[2] Hestenes, D. (2002) Oersted Medal Lecture. http://geocalc.clas.asu.edu/pdf/OerstedMedalLecture.pdf

[3] Arthur, J. (2011) Understanding Geometric Algebra for Electromagnetic Theory. IEEE Press, Hoboken, NJ. https://doi.org/10.1002/9781118078549

[4] Macdonald, A. (2012) Vector and Geometric Calculus. CreateSpace Independent Publishing Platform, Charleston, SC, 63.

[5] Lasenby, A. and Doran, C. (2003) Geometric Algebra for Physicists. Cambridge 
University Press, Cambridge.

[6] Hestenes, D. and Sobcyzk, G. (1984) Clifford Algebra to Geometric Calculus. Reidel Pub. Co., Boston, MA, 242. https://doi.org/10.1007/978-94-009-6292-7

[7] Bechluft-Sach, S. and Samion, E. (2019) Green's Functions, Biot-Savart Operators, and Linking Numbers on Negatively Curved Symmetric Spaces. Journal of Mathematical Physics, 60, Article ID: 111503. https://doi.org/10.1063/1.5109244

[8] Gull, S., Lasenby, A. and Doran, C. (1993) Imaginary Numbers Are Not Real-The Geometric Algebra of Spacetime. Foundations of Physics, 23, 1175-1201. https://doi.org/10.1007/BF01883676

[9] Klingman, E. (2019) A Primordial Space-Time Metric. Prespacetime Journal, 10, 671-680.

[10] Parsley, R.J. (2004) The Biot-Savart Operator and Electrodynamics on Bounded Subdomains of the Three-Sphere. Dissertation, University of Pennsylvania, Philadelphia. 